\title{
Efficacy of Different Bait and Attractant Combinations in Attracting Pumpkin Fruit Fly Zeugodacus (Zeugoodacus) Tau (Walker) (Diptera: Tephritidae)
}

\author{
A. Vasudha*, M. L. Agarwal and M. Sreedhar \\ Department of Entomology, Dr. Rajendra Prasad Central Agricultural University, Pusa \\ (Samastipur) - 848 125, India \\ *Corresponding author
}

\section{Keywords}

Zeugodacus tau, Bait, Attractant,

Protein

hydrolysate, Jaggery, Mollasses, annihilation

\section{Article Info}

Accepted:

18 March 2020

Available Online:

10 April 2020

\section{A B S T R A C T}

An experiment was conducted to determine the efficacy of different bait and attractant combinations in attracting Pumpkin fruit fly Zeugodacus (Zeugodacus) tau from April 2018 to march 2019. Flies were trapped in self-made bottle type traps by using five different combinations $\mathrm{T}_{1}$ (Cue-lure + Dichlorvos + Ethanol), $\mathrm{T}_{2}$ (Protein hydrolysate + Cue-lure + Dichlorvos + Ethanol $), \mathrm{T}_{3}$ (Jaggery + Cuelure + Dichlorvos + Ethanol $), \mathrm{T}_{4}$ (Fruit pulp + Cue-lure + Dichlorvos + Ethanol $)$ and $\mathrm{T}_{5}$ (Molasses + Cue-lure + Dichlorvos + Ethanol). The number of flies trapped was counted at weekly intervals. Maximum flies were annihilated during $20^{\text {th }}$ standard week of 2018 in treatment with protein bait (79.50 flies/ trap/ week) and found it as superior. The rate of male annihilation in treatment with molasses as bait and without bait (Control) did not differed significantly. Mean numbers of flies/ trap annihilated in different treatments were: 400.25 (no bait), 733.25 (protein bait), 503.00 (jaggery as bait), 480.75 (fruit pulp as bait) and 406.25 (molasses as bait), respectively. Efficacy of these treatments in annihilation of male flies with respect to different baits was in the order: protein diet $>$ jaggery $\geq$ fruit pulp > cue-lure $(1 \mathrm{ml}) \geq$ molasses.

\section{Introduction}

A dipteran family, tephritidae, includes the flies often called "fruit flies" and nearly 5,000 described species of tephritid fruit flies are categorized in almost 500 genera. Fruit flies are most economically important pest species in the world, attacking a wide range of fruits and fleshy vegetables throughout tropical and subtropical areas. From India 295 species of fruit flies belonging to 90 genera have been described (Agarwal, 2019) and flies belonging to the genera Bactrocera Macquart and Zeugodacus Hendel (Diptera: Tephritidae: Dacinae: Dacini) are most serious pests causing enormous losses to all kinds of fruits and vegetables. All Dacini members are frugivorous or florivorous and 
about 10 per cent of the 932 currently recognized species are pests of commercial fruits and vegetables (Doorenweerd et al., 2018).

Among tephritids infesting cucurbits, $Z$. cucurbitae, Z. diversa, Z. tau, B. zonata and Dacus ciliatus are the most important and serious pests in India. Pumpkin fruit fly, Zeugodacus (Zeugodacus) tau (Walker) (=Bactrocera (Zeugodacus) tau) damages a number of host plants species belonging to 23 families, but plants belonging to the family Cucurbitaceae are most preferred. The losses caused in fruit crops by insect pests particularly by $Z$. tau have been estimated to be as high as 40 per cent of the production in Indonesia (Hasyim et al., 2004). The array of control methods include insecticide sprays to foliage and soil, bait-sprays, male annihilation techniques, releases of sterilized flies and parasitoids, and cultural controls (Vargas et al., 2015). Efforts have been made to manage fruit flies by using different insecticides, baits either in trap or as cover spray, male annihilation technique (MAT) using attractant and combined use of bait and attractant (Double Attack Method) (Boller, 1983). Areawide control either using MAT or BAT is most desirable for effective management of fruit flies. Taking into consideration the present study of evaluation of efficacies of traps containing baits and attractant combinations in annihilation of males was conducted during 2018-2019.

\section{Materials and Methods}

Studies pertaining to the efficacy of different bait and attractant combinations in attraction of the Pumpkin fruit fly Zeugodacus (Zeugoodacus) tau were conducted in Dr. Rajendra Prasad Central Agriculture University during the year 2018-2019. For annihilation of males, self-made plastic bottle traps (Fig. 1; B) were used (made of 1 liter mineral water bottle). Inside the bottle trap plywood block (Fig. 1; A) was used which was soaked in combination consisting of ethanol + cue-lure + dichlorvos $76 \%$ EC $(6: 4: 1 \mathrm{~V} / \mathrm{V})+$ bait (5 per cent) for trapping of flies. These traps were hanged below the trees at about $2 \mathrm{~m}$ height in places having no direct sunlight. The five different treatments were used in attraction of fruit flies, $\mathrm{T}_{1}$ (Cue-lure + Dichlorvos + Ethanol), $\mathrm{T}_{2}$ (Protein hydrolysate + Cue-lure + Dichlorvos + Ethanol), $\mathrm{T}_{3}$ (Jaggery + Cue-lure + Dichlorvos + Ethanol), $\mathrm{T}_{4}$ (Fruit pulp + Cuelure + Dichlorvos + Ethanol) and $\mathrm{T}_{5}$ (Molasses + Cue-lure + Dichlorvos + Ethanol) (Fig. 1; C). The treatments were replicated four times. Impregnated plywood blocks were replaced at fortnightly interval and the number of flies trapped was counted at weekly intervals.

\section{Results and Discussion}

The data pertaining to efficacy of different bait and attractant combination in attraction of males of Z. tau is depicted in (Table 1\&2, Fig. 2) Males flies were annihilated throughout the experimental period (April, 2018 - March, 2019) except during $1^{\text {st }}$ and $2^{\text {nd }}$ standard weeks of January, 2019 when temperature was very low. Besides, the rate of annihilation was also very low during $51^{\text {st }}$ and $52^{\text {nd }}$ standard weeks of 2018 and $3^{\text {rd }}$ to $5^{\text {th }}$ standard weeks of January, 2019 in all treatments. Maximum flies were annihilated during $20^{\text {th }}$ standard week of 2018 in Treatment $\mathrm{T}_{2}$ (with protein bait) (79.50 flies/ trap/week) followed by during $25^{\text {th }}$ standard week $(56.50$ flies/trap/week) in the same treatment. Besides, the rate of annihilation was comparatively higher during $18^{\text {th }}-20^{\text {th }}$ standard weeks of 2018 , and again during $44^{\text {th }}$ to $48^{\text {th }}$ standard weeks of 2018. An overall perusal of data indicates that in most cases when more numbers of flies were annihilated, the treatments showed significant differences 
and treatment $\mathrm{T}_{2}$ performed best; however, at low population significant difference was not observed with respect to annihilation of adult males of $Z$. tau in different treatments.

The rate of male annihilation in treatment $T_{5}$ (with molasses as bait) and $\mathrm{T}_{1}$ (without bait) did not differed significantly during most standard weeks and it can be predicated that molasses was not effective bait.

The numbers of flies annihilated were high during May and June, 2018 and then a subsequent reduction in rate of annihilation of flies was observed during July to September, 2018. During October and November, 2018 and again an increase in the rate of annihilation was observed and considerably more flies were annihilated; however, the number of flies annihilated during different months did not exhibited a uniform pattern (Table 1).

During December, 2018 and January, 2019 rate of annihilation of flies was very low in all treatments. Maximum number of flies were annihilated in treatment $\mathrm{T}_{2}$ (213.25 flies/ trap/ month) in May, 2018 followed by June (103.25 flies/ trap/ month). During the months of May and June, 2018 and October and November, 2018 the treatment $\mathrm{T}_{2}$ was found significantly superior over others. During July - September, 2018 and December, 2018 the difference in treatments was non-significant.

Minimum flies were annihilated during January, 2019 in all treatments without any significant difference. Since these treatments differed in bait constituents only it may be concluded that the bait enhanced the annihilation rate of flies during its peak population and treatment $T_{2}$ (with protein bait) was significantly superior over others. However, treatments $\mathrm{T}_{5}$ (with molasses as bait) and $T_{1}$ (without bait) were at par and showed no significant difference.

In all treatments, 10094 males of Z. tau were annihilated during the period of experimentation. Mean number of flies/ trap annihilated in $\mathrm{T}_{1}-\mathrm{T}_{5}$ were: $400.25,733.25$, 503.00, 480.75 and 406.25 (Table 2). Maximum flies were annihilated in $\mathrm{T}_{2}$ with protein bait and this treatment was significantly superior over others. Treatments $\mathrm{T}_{3}$ (with jaggery) and $\mathrm{T}_{4}$ (with fruit pulp) were at par and similarly Treatments $T_{1}$ (without bait) and $\mathrm{T}_{5}$ (with molasses) were also at par. Therefore, it can be predicted that molasses was not effective bait in terms of its efficacy in annihilation of males of $Z$. tau while protein bait was most effective.

Duyck et al., (2004) out of six commercially available protein hydrolysates found that Solbait was most effective against male and females of melon fly. Bharathi et al., (2004) reported that that banana and soybean hydrolysate were $85-95 \%$ more attractive to adult $Z$. cucurbitae than fishmeal, beef extract, bread and dog biscuit. Kumar and Agarwal (2005) reported that for trapping males of $Z$. cucurbitae the treatments containing soya powder $2 \mathrm{~g}+$ cue-lure $1 \mathrm{ml}+$ malathion 50 EC $1 \mathrm{ml}$ was most effective. Jhala and Sisodiya (2008) reported that integration of MAT with BAT was the most effective strategy on small ground field as it resulted in the reducing melon fly infestation. Thakur and Gupta (2013) found protein hydrolysate as most attractive for Z. tau and Z. cucurbitae followed by yeast hydrolysate. Ekesi (2016) reviewed the use of protein baits and MAT in fruit fly suppression and suggested that these tactics reduce fruit fly populations by $80-90$ per cent. The results of present experiment also suggest that efficacy of attractant increases when it is mixed with bait and protein bait was found most efficacious in annihilation of males of $Z$. tau. 
Table.1 Mean number of Zeugodacus tau annihilated (male flies/trap/week) in different bait and attractant combinations during 2018-19

\begin{tabular}{|c|c|c|c|c|c|c|c|c|}
\hline \multirow[t]{2}{*}{ Month } & \multirow{2}{*}{$\begin{array}{l}\text { Standard } \\
\text { week }\end{array}$} & \multicolumn{5}{|c|}{ Treatment* } & \multirow[t]{2}{*}{$\operatorname{SEm}( \pm)$} & \multirow{2}{*}{$\begin{array}{c}\text { C.D. } \\
(\mathbf{P}=\mathbf{0 . 0 5})\end{array}$} \\
\hline & & $\mathbf{T}_{1}$ & $\mathbf{T}_{2}$ & $\mathbf{T}_{3}$ & $\mathbf{T}_{4}$ & $\mathbf{T}_{5}$ & & \\
\hline \multirow[t]{4}{*}{ April } & 14 & $\begin{array}{c}5.75 \\
(1.74)\end{array}$ & $\begin{array}{l}17.25 \\
(2.84)\end{array}$ & $\begin{array}{l}10.00 \\
(2.30)\end{array}$ & $\begin{array}{c}9.75 \\
(2.27)\end{array}$ & $\begin{array}{c}6.75 \\
(1.90)\end{array}$ & 0.725 & $\begin{array}{l}2.257 \\
(0.81)\end{array}$ \\
\hline & 15 & $\begin{array}{c}5.75 \\
(1.74)\end{array}$ & $\begin{array}{l}18.00 \\
(2.89)\end{array}$ & $\begin{array}{l}11.00 \\
(2.39)\end{array}$ & $\begin{array}{l}10.75 \\
(2.37)\end{array}$ & $\begin{array}{c}8.75 \\
(2.16)\end{array}$ & $\begin{array}{l}1.226 \\
((0.19)\end{array}$ & $\begin{array}{l}3.818 \\
(1.33)\end{array}$ \\
\hline & 16 & $\begin{array}{c}6.25 \\
(1.83)\end{array}$ & $\begin{array}{l}16.75 \\
(2.81)\end{array}$ & $\begin{array}{l}12.75 \\
(2.54)\end{array}$ & $\begin{array}{l}11.00 \\
(2.39)\end{array}$ & $\begin{array}{c}8.25 \\
(2.11)\end{array}$ & $\begin{array}{l}1.213 \\
(0.19)\end{array}$ & $\begin{array}{l}3.778 \\
(1.32)\end{array}$ \\
\hline & 17 & $\begin{array}{c}7.75 \\
(2.04)\end{array}$ & $\begin{array}{l}19.00 \\
(2.94)\end{array}$ & $\begin{array}{l}13.00 \\
(2.56)\end{array}$ & $\begin{array}{l}12.25 \\
(2.50)\end{array}$ & $\begin{array}{l}11.00 \\
(2.39)\end{array}$ & 0.932 & $\begin{array}{l}2.904 \\
(1.06)\end{array}$ \\
\hline Total & & $\begin{array}{l}25.50 \\
(3.23)\end{array}$ & $\begin{array}{c}71.00 \\
(41.26)\end{array}$ & $\begin{array}{l}46.75 \\
(3.84)\end{array}$ & $\begin{array}{l}43.75 \\
(3.77)\end{array}$ & $\begin{array}{l}36.00 \\
(3.58)\end{array}$ & $\begin{array}{l}2.070 \\
(0.72)\end{array}$ & $\begin{array}{l}6.449 \\
(1.86)\end{array}$ \\
\hline \multirow[t]{5}{*}{ May } & 18 & $\begin{array}{l}12.50 \\
(2.52)\end{array}$ & $\begin{array}{l}47.75 \\
(3.86)\end{array}$ & $\begin{array}{l}17.75 \\
(2.87)\end{array}$ & $\begin{array}{l}13.50 \\
(2.60)\end{array}$ & $\begin{array}{l}13.25 \\
(2.58)\end{array}$ & $\begin{array}{l}1.536 \\
(0.42)\end{array}$ & $\begin{array}{l}4.786 \\
(1.56)\end{array}$ \\
\hline & 19 & $\begin{array}{l}22.75 \\
(3.12)\end{array}$ & $\begin{array}{l}52.00 \\
(3.95)\end{array}$ & $\begin{array}{l}27.50 \\
(3.31)\end{array}$ & $\begin{array}{l}19.00 \\
(2.94)\end{array}$ & $\begin{array}{l}13.50 \\
(2.60)\end{array}$ & $\begin{array}{l}1.352 \\
(0.30)\end{array}$ & $\begin{array}{l}4.214 \\
(1.43)\end{array}$ \\
\hline & 20 & $\begin{array}{l}35.75 \\
(3.57)\end{array}$ & $\begin{array}{l}79.50 \\
(4.37)\end{array}$ & $\begin{array}{l}51.50 \\
(3.94)\end{array}$ & $\begin{array}{l}42.25 \\
(3.74)\end{array}$ & $\begin{array}{l}26.50 \\
(3.27)\end{array}$ & $\begin{array}{l}4.282 \\
(1.45)\end{array}$ & $\begin{array}{l}13.339 \\
(2.59)\end{array}$ \\
\hline & 21 & $\begin{array}{l}19.25 \\
(2.95)\end{array}$ & $\begin{array}{l}17.50 \\
(2.86)\end{array}$ & $\begin{array}{l}15.25 \\
(2.72)\end{array}$ & $\begin{array}{l}10.25 \\
(2.32)\end{array}$ & $\begin{array}{l}12.00 \\
(2.48)\end{array}$ & $\begin{array}{l}2.744 \\
(1.00)\end{array}$ & NS \\
\hline & 22 & $\begin{array}{l}19.00 \\
(2.94)\end{array}$ & $\begin{array}{l}16.50 \\
(2.80)\end{array}$ & $\begin{array}{l}13.75 \\
(2.62)\end{array}$ & $\begin{array}{l}24.00 \\
(3.17)\end{array}$ & $\begin{array}{l}12.25 \\
(2.50)\end{array}$ & $\begin{array}{c}2.28 \\
(0.82)\end{array}$ & $\begin{array}{l}7.103 \\
(1.96)\end{array}$ \\
\hline Total & & $\begin{array}{l}109.25 \\
(4.69)\end{array}$ & $\begin{array}{l}213.25 \\
(5.36)\end{array}$ & $\begin{array}{l}125.75 \\
(4.83)\end{array}$ & $\begin{array}{l}109.00 \\
(4.69)\end{array}$ & $\begin{array}{l}77.50 \\
(4.35)\end{array}$ & $\begin{array}{l}6.883 \\
(1.92)\end{array}$ & $\begin{array}{l}21.445 \\
(3.06)\end{array}$ \\
\hline \multirow[t]{4}{*}{ June } & 23 & $\begin{array}{l}20.50 \\
(3.02)\end{array}$ & $\begin{array}{l}22.00 \\
(3.09)\end{array}$ & $\begin{array}{l}19.50 \\
(2.97)\end{array}$ & $\begin{array}{l}13.25 \\
(2.58)\end{array}$ & $\begin{array}{l}15.00 \\
(2.70)\end{array}$ & $\begin{array}{l}2.261 \\
(0.85)\end{array}$ & NS \\
\hline & 24 & $\begin{array}{l}10.00 \\
(2.30)\end{array}$ & $\begin{array}{l}11.50 \\
(2.44)\end{array}$ & $\begin{array}{c}5.50 \\
(1.70)\end{array}$ & $\begin{array}{l}16.50 \\
(2.80)\end{array}$ & $\begin{array}{c}8.00 \\
(2.07)\end{array}$ & $\begin{array}{c}1.63 \\
(0.48)\end{array}$ & $\begin{array}{c}5.08 \\
(1.62)\end{array}$ \\
\hline & 25 & $\begin{array}{l}15.25 \\
(2.72)\end{array}$ & $\begin{array}{l}56.50 \\
(4.03\end{array}$ & $\begin{array}{l}18.50 \\
(2.91)\end{array}$ & $\begin{array}{l}37.50 \\
(3.62)\end{array}$ & $\begin{array}{l}20.00 \\
(2.99)\end{array}$ & $\begin{array}{l}4.204 \\
(1.43)\end{array}$ & $\begin{array}{l}13.096 \\
(2.57)\end{array}$ \\
\hline & 26 & $\begin{array}{c}8.75 \\
(2.16)\end{array}$ & $\begin{array}{l}13.25 \\
(2.58)\end{array}$ & $\begin{array}{l}14.00 \\
(2.63)\end{array}$ & $\begin{array}{l}17.25 \\
(2.84)\end{array}$ & $\begin{array}{l}13.50 \\
(2.60)\end{array}$ & $\begin{array}{l}1.796 \\
(0.58)\end{array}$ & NS \\
\hline Total & & $\begin{array}{l}54.50 \\
(3.99)\end{array}$ & $\begin{array}{l}103.25 \\
(4.63)\end{array}$ & $\begin{array}{l}57.50 \\
(4.05)\end{array}$ & $\begin{array}{l}84.50 \\
(4.43)\end{array}$ & $\begin{array}{l}56.50 \\
(4.03)\end{array}$ & $\begin{array}{l}6.061 \\
(1.80)\end{array}$ & $\begin{array}{c}18.900 \\
(2.93)\end{array}$ \\
\hline \multirow[t]{4}{*}{ July } & 27 & $\begin{array}{c}7.25 \\
(1.98)\end{array}$ & $\begin{array}{l}12.25 \\
(2.50)\end{array}$ & $\begin{array}{l}12.50 \\
(2.52)\end{array}$ & $\begin{array}{l}18.00 \\
(2.89)\end{array}$ & $\begin{array}{c}7.00 \\
(1.94)\end{array}$ & $\begin{array}{l}1.646 \\
(0.49)\end{array}$ & $\begin{array}{l}5.129 \\
(1.63)\end{array}$ \\
\hline & 28 & $\begin{array}{c}7.50 \\
(2.01)\end{array}$ & $\begin{array}{l}10.50 \\
(2.35)\end{array}$ & $\begin{array}{l}10.25 \\
(2.32)\end{array}$ & $\begin{array}{c}9.50 \\
(2.25)\end{array}$ & $\begin{array}{l}11.50 \\
(2.44)\end{array}$ & $\begin{array}{l}1.199 \\
(0.18)\end{array}$ & NS \\
\hline & 29 & $\begin{array}{l}5.00 \\
(1.6)\end{array}$ & $\begin{array}{c}6.25 \\
(1.83)\end{array}$ & $\begin{array}{c}4.00 \\
(1.38)\end{array}$ & $\begin{array}{c}5.00 \\
(1.60)\end{array}$ & $\begin{array}{c}7.25 \\
(1.98)\end{array}$ & $\begin{array}{l}1.157 \\
(0.14)\end{array}$ & NS \\
\hline & 30 & $\begin{array}{c}1.50 \\
(0.40)\end{array}$ & $\begin{array}{c}4.25 \\
(1.44)\end{array}$ & $\begin{array}{c}3.25 \\
(1.17)\end{array}$ & $\begin{array}{c}2.00 \\
(0.69)\end{array}$ & $\begin{array}{c}4.50 \\
(1.50)\end{array}$ & $\begin{array}{l}1.148 \\
(0.14)\end{array}$ & NS \\
\hline Total & & $\begin{array}{l}21.25 \\
(3.05)\end{array}$ & $\begin{array}{l}33.25 \\
(3.50)\end{array}$ & $\begin{array}{l}30.00 \\
(3.40)\end{array}$ & $\begin{array}{l}34.50 \\
(3.54)\end{array}$ & $\begin{array}{l}30.25 \\
(3.40)\end{array}$ & $\begin{array}{l}4.123 \\
(1.41)\end{array}$ & NS \\
\hline \multirow[t]{3}{*}{ August } & 31 & $\begin{array}{c}3.50 \\
(1.25)\end{array}$ & $\begin{array}{c}2.75 \\
(1.01)\end{array}$ & $\begin{array}{c}1.50 \\
(0.40)\end{array}$ & $\begin{array}{c}3.00 \\
(1.09)\end{array}$ & $\begin{array}{c}3.75 \\
(1.32)\end{array}$ & 0.766 & NS \\
\hline & 32 & $\begin{array}{c}6.50 \\
(1.87)\end{array}$ & $\begin{array}{c}6.75 \\
(1.90)\end{array}$ & $\begin{array}{l}10.75 \\
(2.37)\end{array}$ & $\begin{array}{l}12.75 \\
(2.54)\end{array}$ & $\begin{array}{c}5.75 \\
(1.74)\end{array}$ & $\begin{array}{c}1.43 \\
(0.35)\end{array}$ & $\begin{array}{l}4.456 \\
(1.49)\end{array}$ \\
\hline & 33 & $\begin{array}{c}8.25 \\
(2.11)\end{array}$ & $\begin{array}{c}5.25 \\
(1.65)\end{array}$ & $\begin{array}{c}3.50 \\
(1.25)\end{array}$ & $\begin{array}{c}3.75 \\
(1.32)\end{array}$ & $\begin{array}{c}3.75 \\
(1.32)\end{array}$ & 0.942 & $\begin{array}{l}2.935 \\
(1.07)\end{array}$ \\
\hline
\end{tabular}




\begin{tabular}{|c|c|c|c|c|c|c|c|c|}
\hline & 34 & $\begin{array}{c}6.25 \\
(1.83)\end{array}$ & $\begin{array}{c}9.50 \\
(2.25)\end{array}$ & $\begin{array}{c}6.00 \\
(1.79)\end{array}$ & $\begin{array}{c}5.50 \\
(1.70)\end{array}$ & $\begin{array}{c}7.50 \\
(2.01)\end{array}$ & $\begin{array}{l}1.332 \\
(0.28)\end{array}$ & NS \\
\hline & 35 & $\begin{array}{c}7.50 \\
(2.01)\end{array}$ & $\begin{array}{c}8.75 \\
(2.16)\end{array}$ & $\begin{array}{c}9.75 \\
(2.27)\end{array}$ & $\begin{array}{l}10.00 \\
(2.30)\end{array}$ & $\begin{array}{c}5.75 \\
(1.74)\end{array}$ & $\begin{array}{c}1.02 \\
(0.01)\end{array}$ & NS \\
\hline Total & & $\begin{array}{l}32.00 \\
(3.46)\end{array}$ & $\begin{array}{l}33.00 \\
(3.49)\end{array}$ & $\begin{array}{l}31.50 \\
(3.44)\end{array}$ & $\begin{array}{l}35.00 \\
(3.55)\end{array}$ & $\begin{array}{l}26.50 \\
(3.27)\end{array}$ & $\begin{array}{l}3.208 \\
(1.16)\end{array}$ & NS \\
\hline \multirow[t]{4}{*}{ September } & 36 & $\begin{array}{c}6.00 \\
(1.79)\end{array}$ & $\begin{array}{c}9.00 \\
(2.19)\end{array}$ & $\begin{array}{l}11.75 \\
(2.46)\end{array}$ & $\begin{array}{c}5.25 \\
(1.65)\end{array}$ & $\begin{array}{c}5.75 \\
(1.74)\end{array}$ & $\begin{array}{l}1.073 \\
(0.07)\end{array}$ & $\begin{array}{l}3.344 \\
(1.20)\end{array}$ \\
\hline & 37 & $\begin{array}{c}6.00 \\
(1.79)\end{array}$ & $\begin{array}{c}5.25 \\
(1.65)\end{array}$ & $\begin{array}{c}7.50 \\
(2.01)\end{array}$ & $\begin{array}{c}8.00 \\
(2.07)\end{array}$ & $\begin{array}{c}5.00 \\
(1.60)\end{array}$ & $\begin{array}{l}2.064 \\
(0.72)\end{array}$ & NS \\
\hline & 38 & $\begin{array}{c}6.75 \\
(1.90)\end{array}$ & $\begin{array}{c}6.25 \\
(1.83)\end{array}$ & $\begin{array}{c}8.25 \\
(2.11)\end{array}$ & $\begin{array}{c}8.25 \\
(2.11)\end{array}$ & $\begin{array}{c}6.75 \\
(1.90)\end{array}$ & 0.851 & NS \\
\hline & 39 & $\begin{array}{c}5.25 \\
(1.65)\end{array}$ & $\begin{array}{c}9.00 \\
(2.19)\end{array}$ & $\begin{array}{c}8.50 \\
(2.14)\end{array}$ & $\begin{array}{c}5.00 \\
(1.60)\end{array}$ & $\begin{array}{c}8.75 \\
(2.16)\end{array}$ & $\begin{array}{c}1.186 \\
(0.170)\end{array}$ & NS \\
\hline Total & & $\begin{array}{l}24.00 \\
(3.17)\end{array}$ & $\begin{array}{l}29.50 \\
(3.38)\end{array}$ & $\begin{array}{l}36.00 \\
(3.58)\end{array}$ & $\begin{array}{l}26.50 \\
(3.27)\end{array}$ & $\begin{array}{l}26.25 \\
(3.26)\end{array}$ & $\begin{array}{l}2.971 \\
(1.08)\end{array}$ & NS \\
\hline \multirow[t]{4}{*}{ October } & 40 & $\begin{array}{l}10.00 \\
(2.30)\end{array}$ & $\begin{array}{l}13.25 \\
(2.58)\end{array}$ & $\begin{array}{l}10.50 \\
(2.35)\end{array}$ & $\begin{array}{c}8.75 \\
(2.16)\end{array}$ & $\begin{array}{c}8.00 \\
(2.07)\end{array}$ & 0.956 & $\begin{array}{c}2.9 \\
(1.06)\end{array}$ \\
\hline & 41 & $\begin{array}{c}5.50 \\
(1.70)\end{array}$ & $\begin{array}{c}9.00 \\
(2.19)\end{array}$ & $\begin{array}{c}9.25 \\
(2.22)\end{array}$ & $\begin{array}{c}5.50 \\
(1.70)\end{array}$ & $\begin{array}{c}5.00 \\
(1.60)\end{array}$ & 0.985 & 3.07 \\
\hline & 42 & $\begin{array}{c}6.50 \\
(1.87)\end{array}$ & $\begin{array}{c}9.50 \\
(2.25)\end{array}$ & $\begin{array}{c}6.25 \\
(1.83)\end{array}$ & $\begin{array}{c}4.50 \\
(1.50)\end{array}$ & $\begin{array}{c}8.25 \\
(2.11)\end{array}$ & $\begin{array}{l}1.536 \\
(0.43)\end{array}$ & $\begin{array}{l}4.786 \\
(1.56)\end{array}$ \\
\hline & 43 & $\begin{array}{c}9.25 \\
(2.22)\end{array}$ & $\begin{array}{l}21.75 \\
(3.07)\end{array}$ & $\begin{array}{l}14.00 \\
(2.63)\end{array}$ & $\begin{array}{l}12.00 \\
(2.48)\end{array}$ & $\begin{array}{l}13.50 \\
(2.60)\end{array}$ & $\begin{array}{l}1.352 \\
(0.30)\end{array}$ & $\begin{array}{l}4.214 \\
(1.43)\end{array}$ \\
\hline Total & & $\begin{array}{l}31.25 \\
(3.44)\end{array}$ & $\begin{array}{l}53.50 \\
(3.97)\end{array}$ & $\begin{array}{l}40.00 \\
(3.68)\end{array}$ & $\begin{array}{l}30.75 \\
(3.42)\end{array}$ & $\begin{array}{l}34.75 \\
(3.54)\end{array}$ & $\begin{array}{l}3.038 \\
(1.18)\end{array}$ & $\begin{array}{l}9.446 \\
(2.24)\end{array}$ \\
\hline \multirow[t]{5}{*}{ November } & 44 & $\begin{array}{l}10.25 \\
(2.32)\end{array}$ & $\begin{array}{l}21.00 \\
(3.04)\end{array}$ & $\begin{array}{l}17.50 \\
(2.86)\end{array}$ & $\begin{array}{l}12.50 \\
(2.52)\end{array}$ & $\begin{array}{l}12.50 \\
(2.52)\end{array}$ & $\begin{array}{l}4.282 \\
(1.45)\end{array}$ & $\begin{array}{c}13.339 \\
(2.59)\end{array}$ \\
\hline & 45 & $\begin{array}{l}13.25 \\
(2.58)\end{array}$ & $\begin{array}{l}11.25 \\
(2.42)\end{array}$ & $\begin{array}{l}10.50 \\
(2.35)\end{array}$ & $\begin{array}{c}8.25 \\
(2.11)\end{array}$ & $\begin{array}{l}10.50 \\
(2.35)\end{array}$ & $\begin{array}{l}2.744 \\
(1.00)\end{array}$ & NS \\
\hline & 46 & $\begin{array}{l}14.50 \\
(2.67)\end{array}$ & $\begin{array}{l}20.25 \\
(3.00)\end{array}$ & $\begin{array}{l}10.50 \\
(2.35)\end{array}$ & $\begin{array}{l}10.00 \\
(2.30\end{array}$ & $\begin{array}{l}13.75 \\
(2.62)\end{array}$ & $\begin{array}{c}2.28 \\
(0.82)\end{array}$ & $\begin{array}{c}7.103 \\
(1.96)\end{array}$ \\
\hline & 47 & $\begin{array}{l}12.25 \\
(2.50)\end{array}$ & $\begin{array}{l}16.25 \\
(2.78)\end{array}$ & $\begin{array}{l}10.75 \\
(2.37)\end{array}$ & $\begin{array}{l}10.00 \\
(2.30)\end{array}$ & $\begin{array}{l}13.75 \\
(2.62)\end{array}$ & $\begin{array}{l}2.261 \\
(0.85)\end{array}$ & NS \\
\hline & 48 & $\begin{array}{l}10.25 \\
(2.32)\end{array}$ & $\begin{array}{l}12.00 \\
(2.48)\end{array}$ & $\begin{array}{c}6.25 \\
(1.83)\end{array}$ & $\begin{array}{c}9.50 \\
(2.25)\end{array}$ & $\begin{array}{l}11.50 \\
(2.44)\end{array}$ & $\begin{array}{c}1.63 \\
(0.49)\end{array}$ & $\begin{array}{c}5.08 \\
(1.62)\end{array}$ \\
\hline Total & & $\begin{array}{l}60.50 \\
(4.10)\end{array}$ & $\begin{array}{l}80.75 \\
(4.39)\end{array}$ & $\begin{array}{l}55.50 \\
(4.01)\end{array}$ & $\begin{array}{l}50.25 \\
(3.91)\end{array}$ & $\begin{array}{c}62.00 \\
(44.27)\end{array}$ & $\begin{array}{c}4.670 \\
(1.054)\end{array}$ & $\begin{array}{c}14.548 \\
(2.67)\end{array}$ \\
\hline \multirow[t]{4}{*}{ December } & 49 & $\begin{array}{c}5.75 \\
(1.74)\end{array}$ & $\begin{array}{c}8.00 \\
(2.07)\end{array}$ & $\begin{array}{c}5.00 \\
(1.60)\end{array}$ & $\begin{array}{c}3.50 \\
(1.25)\end{array}$ & $\begin{array}{c}5.00 \\
(1.60)\end{array}$ & $\begin{array}{l}4.204 \\
(1.44)\end{array}$ & $\begin{array}{c}13.096 \\
(2.57)\end{array}$ \\
\hline & 50 & $\begin{array}{c}1.50 \\
(0.40)\end{array}$ & $\begin{array}{c}4.75 \\
(1.55)\end{array}$ & $\begin{array}{c}6.00 \\
(1.79)\end{array}$ & $\begin{array}{c}4.75 \\
(1.55)\end{array}$ & $\begin{array}{c}5.50 \\
(1.07)\end{array}$ & $\begin{array}{l}1.796 \\
(0.58)\end{array}$ & NS \\
\hline & 51 & $\begin{array}{c}1.00 \\
(0.00)\end{array}$ & $\begin{array}{c}2.00 \\
(0.693)\end{array}$ & $\begin{array}{c}1.25 \\
(0.223)\end{array}$ & $\begin{array}{c}1.00 \\
(0.00)\end{array}$ & 0.75 & $\begin{array}{l}1.646 \\
(0.50)\end{array}$ & $\begin{array}{l}5.129 \\
(1.63)\end{array}$ \\
\hline & 52 & $\begin{array}{c}1.00 \\
(0.00)\end{array}$ & $\begin{array}{c}1.00 \\
(0.00)\end{array}$ & $\begin{array}{c}1.25 \\
(0.22)\end{array}$ & $\begin{array}{c}1.25 \\
(0.22)\end{array}$ & $\begin{array}{c}1.00 \\
(0.00)\end{array}$ & $\begin{array}{l}1.199 \\
(0.18)\end{array}$ & NS \\
\hline Total & & $\begin{array}{c}9.25 \\
(2.22)\end{array}$ & $\begin{array}{l}15.75 \\
(2.75)\end{array}$ & $\begin{array}{l}13.50 \\
(2.60)\end{array}$ & $\begin{array}{l}10.50 \\
(2.35)\end{array}$ & $\begin{array}{l}12.25 \\
(2.50)\end{array}$ & $\begin{array}{l}2.078 \\
(0.73)\end{array}$ & NS \\
\hline \multirow[t]{3}{*}{ January } & 1 & 0.00 & 0.00 & 0.00 & 0.00 & 0.00 & - & - \\
\hline & 2 & 0.00 & 0.00 & 0.00 & 0.00 & 0.00 & - & - \\
\hline & 3 & 0.25 & $\begin{array}{c}1.50 \\
(0.40)\end{array}$ & 0.25 & 0.25 & 0.25 & 0.359 & NS \\
\hline
\end{tabular}




\begin{tabular}{|c|c|c|c|c|c|c|c|c|}
\hline & 4 & 0.75 & 0.75 & 0.50 & 0.75 & 0.75 & 0.423 & NS \\
\hline Total & & $\begin{array}{c}1.00 \\
(0.00)\end{array}$ & 2.25 & 0.75 & $\begin{array}{l}1.00 \\
(0.0)\end{array}$ & $\begin{array}{c}1.00 \\
(0.00)\end{array}$ & 0.454 & NS \\
\hline \multirow[t]{4}{*}{ February } & 5 & 0.50 & $\begin{array}{c}1.50 \\
(0.40)\end{array}$ & 0.50 & 0.75 & $\begin{array}{c}1.00 \\
(0.00)\end{array}$ & 0.540 & NS \\
\hline & 6 & $\begin{array}{c}1.50 \\
(0.40)\end{array}$ & $\begin{array}{c}4.50 \\
(1.50)\end{array}$ & $\begin{array}{c}3.25 \\
(1.17)\end{array}$ & $\begin{array}{c}3.00 \\
(1.09)\end{array}$ & $\begin{array}{c}3.75 \\
(1.32)\end{array}$ & 0.339 & $\begin{array}{l}1.055 \\
(0.04)\end{array}$ \\
\hline & 7 & $\begin{array}{c}2.00 \\
(0.69)\end{array}$ & $\begin{array}{c}7.50 \\
(2.01)\end{array}$ & $\begin{array}{c}4.25 \\
(1.44)\end{array}$ & $\begin{array}{c}3.25 \\
(1.17)\end{array}$ & $\begin{array}{c}2.50 \\
(0.91)\end{array}$ & 0.703 & $\begin{array}{l}2.189 \\
(0.78)\end{array}$ \\
\hline & 8 & $\begin{array}{c}2.75 \\
(1.01)\end{array}$ & $\begin{array}{l}13.75 \\
(2.62)\end{array}$ & $\begin{array}{r}8.75 \\
(2.16)\end{array}$ & $\begin{array}{c}5.50 \\
(1.70)\end{array}$ & $\begin{array}{c}3.00 \\
(1.09)\end{array}$ & $\begin{array}{l}1.048 \\
(0.04)\end{array}$ & $\begin{array}{l}3.264 \\
(1.18)\end{array}$ \\
\hline Total & & $\begin{array}{c}6.75 \\
(1.90)\end{array}$ & $\begin{array}{l}27.25 \\
(3.30)\end{array}$ & $\begin{array}{l}16.75 \\
(2.81)\end{array}$ & $\begin{array}{l}12.50 \\
(2.52)\end{array}$ & $\begin{array}{c}9.75 \\
(2.27)\end{array}$ & 0.173 & $\begin{array}{l}3.653 \\
(1.27)\end{array}$ \\
\hline \multirow[t]{5}{*}{ March } & 9 & $\begin{array}{c}4.75 \\
(1.55)\end{array}$ & $\begin{array}{l}12.75 \\
(2.54)\end{array}$ & $\begin{array}{c}8.25 \\
(2.11)\end{array}$ & $\begin{array}{c}7.50 \\
(2.01)\end{array}$ & $\begin{array}{c}6.00 \\
(1.79)\end{array}$ & $\begin{array}{c}1.154 \\
(0.139)\end{array}$ & $\begin{array}{l}3.595 \\
(1.28)\end{array}$ \\
\hline & 10 & $\begin{array}{c}4.75 \\
(1.55)\end{array}$ & $\begin{array}{l}15.50 \\
(2.74)\end{array}$ & $\begin{array}{c}7.00 \\
(1.94)\end{array}$ & $\begin{array}{c}8.50 \\
(2.14)\end{array}$ & $\begin{array}{c}6.75 \\
(1.90)\end{array}$ & 0.808 & $\begin{array}{l}2.516 \\
(0.92)\end{array}$ \\
\hline & 11 & $\begin{array}{c}5.25 \\
(1.65)\end{array}$ & $\begin{array}{l}16.25 \\
(2.78)\end{array}$ & $\begin{array}{c}9.00 \\
(2.19)\end{array}$ & $\begin{array}{c}7.50 \\
(2.01)\end{array}$ & $\begin{array}{c}9.25 \\
(2.22)\end{array}$ & 0.907 & $\begin{array}{l}2.826 \\
(1.03)\end{array}$ \\
\hline & 12 & $\begin{array}{c}4.25 \\
(1.44)\end{array}$ & $\begin{array}{l}13.50 \\
(2.60)\end{array}$ & $\begin{array}{l}12.00 \\
(2.48)\end{array}$ & $\begin{array}{l}11.00 \\
(2.39)\end{array}$ & $\begin{array}{c}7.25 \\
(1.98)\end{array}$ & 0.872 & $\begin{array}{l}2.717 \\
(0.99)\end{array}$ \\
\hline & 13 & $\begin{array}{c}5.75 \\
(1.74)\end{array}$ & $\begin{array}{l}16.25 \\
(2.78)\end{array}$ & $\begin{array}{l}11.75 \\
(2.46)\end{array}$ & $\begin{array}{c}9.25 \\
(2.22)\end{array}$ & $\begin{array}{c}9.00 \\
(2.19)\end{array}$ & $\begin{array}{l}1.283 \\
(0.24)\end{array}$ & $\begin{array}{l}3.997 \\
(1.38)\end{array}$ \\
\hline Total & & $\begin{array}{l}25.00 \\
(3.21)\end{array}$ & $\begin{array}{l}70.50 \\
(4.25)\end{array}$ & $\begin{array}{l}49.00 \\
(3.89)\end{array}$ & $\begin{array}{l}40.25 \\
(3.69)\end{array}$ & $\begin{array}{l}35.25 \\
(3.56)\end{array}$ & $\begin{array}{l}1.902 \\
(0.64)\end{array}$ & $\begin{array}{l}5.926 \\
(1.77)\end{array}$ \\
\hline
\end{tabular}

* Mean of four replications; Figures in parentheses are loge transformed values; NS = Non-significant T1 Control $=[$ ethanol + cue-lure + dichlorvos $(6: 4: 1 \mathrm{~V} / \mathrm{V})]$ $\mathrm{T} 2=[($ Protein hydrolysate $5 \%)+$ ethanol + cue-lure + dichlorvos $(6: 4: 1 \mathrm{~V} / \mathrm{V})]$

$\mathrm{T} 3=[($ Jaggery $5 \%)+$ ethanol + cue-lure + dichlorvos $(6: 4: 1 \mathrm{~V} / \mathrm{V})]$

$\mathrm{T} 4=[($ Fruit pulp 5\%) + ethanol + cue-lure + dichlorvos $(6: 4: 1 \mathrm{~V} / \mathrm{V})]$

$\mathrm{T} 5=[($ Molasses $5 \%)+$ ethanol + cue-lure + dichlorvos $(6: 4: 1 \mathrm{~V} / \mathrm{V})]$

Table.2 Total number of male flies of Zeugodacus tau annihilated in different bait and attractant combinations during 2018-19

\begin{tabular}{|c|c|c|c|c|c|c|}
\hline \multirow[t]{2}{*}{ Treatment } & \multicolumn{4}{|c|}{ Number of flies } & \multirow[t]{2}{*}{ Total } & \multirow{2}{*}{$\begin{array}{c}\text { Mean } \\
\text { (Flies/trap) }\end{array}$} \\
\hline & $\mathrm{R}_{\mathrm{I}}$ & $\mathrm{R}_{\mathrm{II}}$ & $\mathrm{R}_{\mathrm{III}}$ & $\mathrm{R}_{\mathrm{IV}}$ & & \\
\hline $\mathbf{T}_{1}$ & 454.00 & 419.00 & 365.00 & 363.00 & 1601.00 & $\begin{array}{c}400.25 \\
(5.99)\end{array}$ \\
\hline $\mathbf{T}_{2}$ & 845.00 & 742.00 & 708.00 & 638.00 & 2933.00 & $\begin{array}{l}733.25 \\
(6.59)\end{array}$ \\
\hline $\mathbf{T}_{\mathbf{3}}$ & 533.00 & 543.00 & 484.00 & 452.00 & 2012.00 & $\begin{array}{c}503.00 \\
(6.22)\end{array}$ \\
\hline $\mathbf{T}_{4}$ & 558.00 & 495.00 & 434.00 & 436.00 & 1923.00 & $\begin{array}{c}480.75 \\
(6.17)\end{array}$ \\
\hline $\mathbf{T}_{5}$ & 438.00 & 403.00 & 393.00 & 391.00 & 1625.00 & $\begin{array}{c}406.25 \\
(6.00)\end{array}$ \\
\hline Total & 2828.00 & 2602.00 & 2384.00 & 2280.00 & 10094.00 & \\
\hline
\end{tabular}

Figures in parentheses are $\log _{\mathrm{e}}$ transformed values; $\quad$ C.D. $(\mathrm{P}=0.05)=44.665(3.79), \operatorname{SEm}(( \pm)=14.337(2.66)$ 

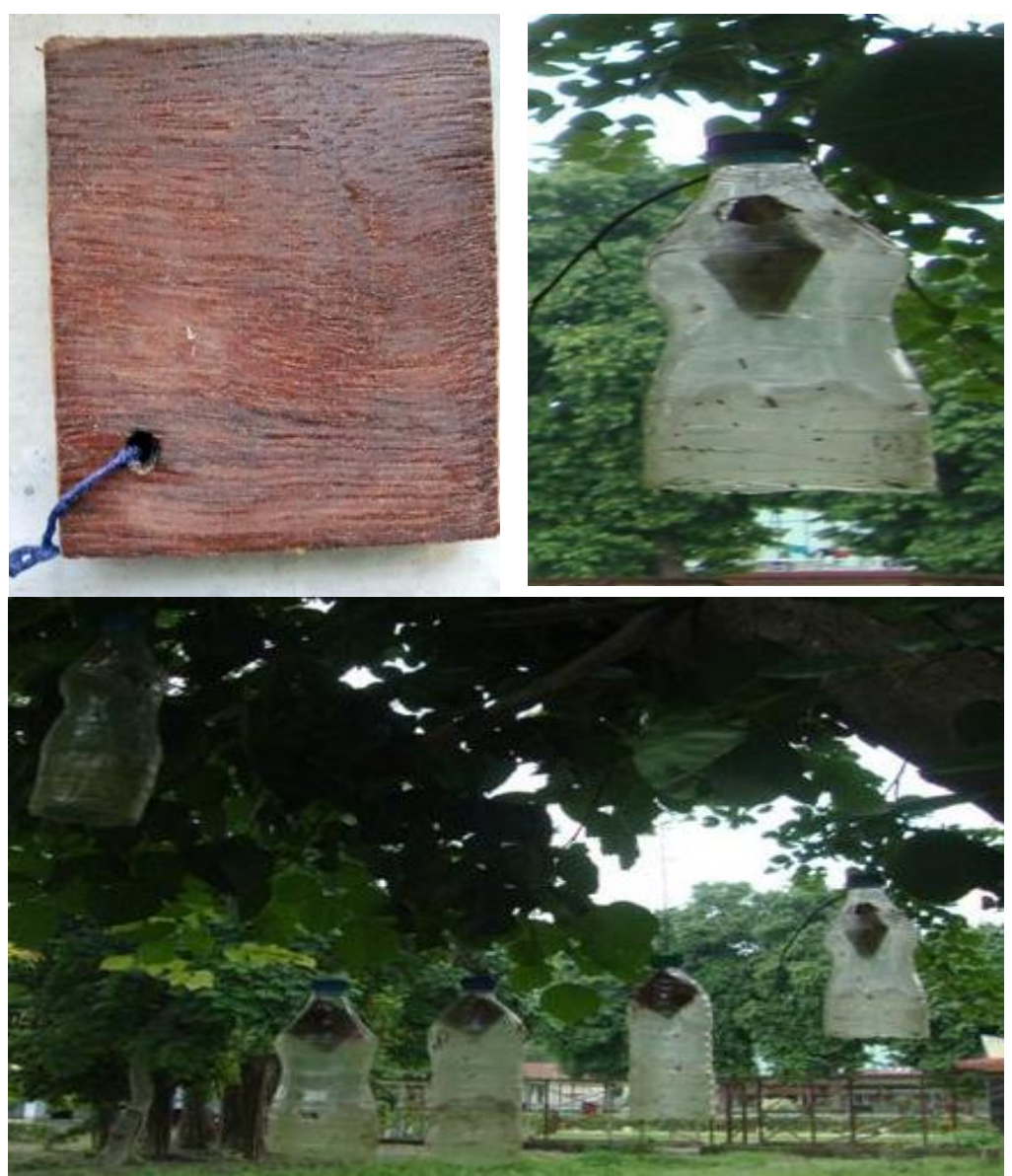

Fig.1 A) Plywood block B) Self-made bottle trap C) Bottle traps with different bait and attractant combinations

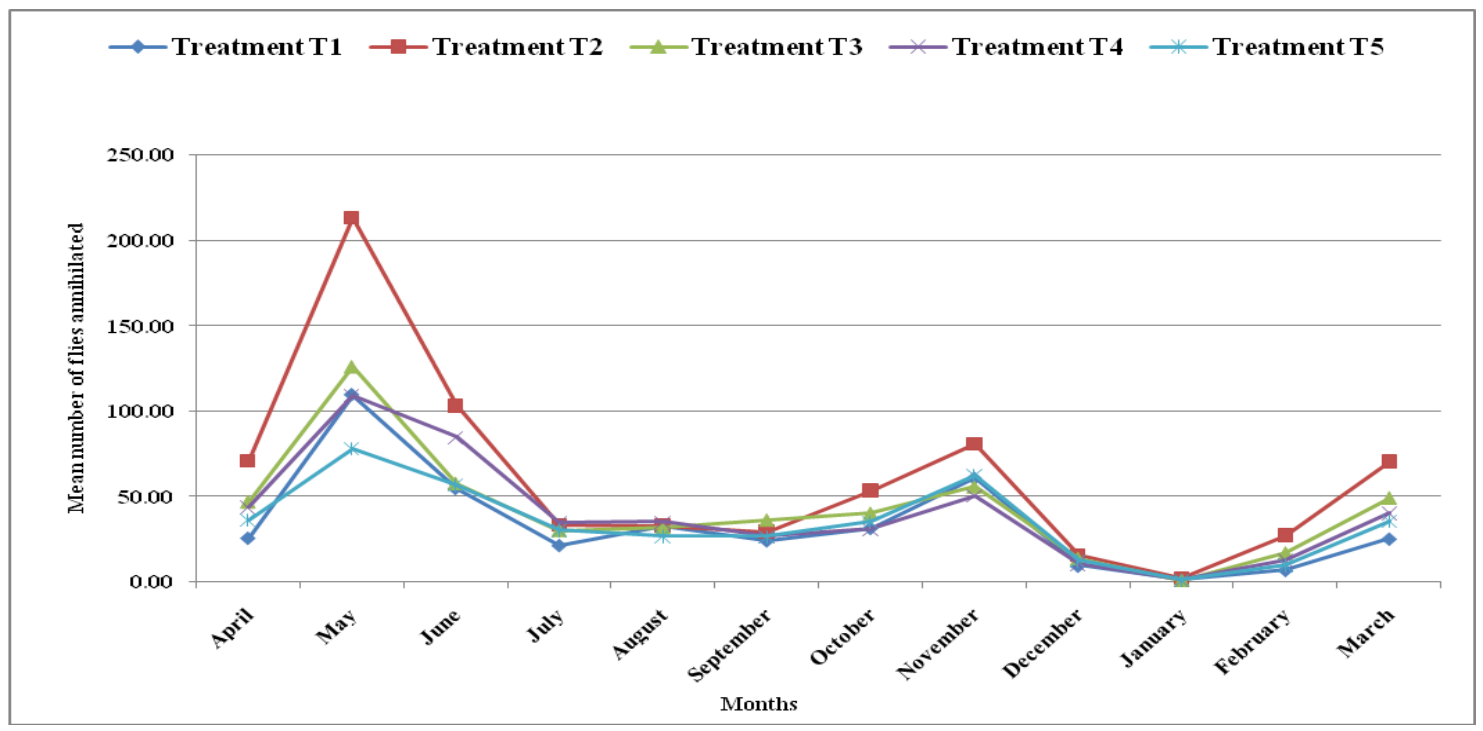

Fig.2 Mean number of Zeugodacus tau trapped (males/trap/month) in different bait and attractant combinations during 2018-19 
Mean numbers of flies/ trap annihilated in different treatments were: 400.25 (no bait), 733.25 (protein bait), 503.00 (jaggery as bait), 480.75 (fruit pulp as bait) and 406.25 molasses as bait), respectively. Efficacy of these treatments in annihilation of male flies with respect to different baits was in the order: protein diet $>$ jaggery $\geq$ fruit pulp $>$ cue-lure $(1 \mathrm{ml}) \geq$ molasses. Therefore, it can be predicted that molasses was not effective bait in terms of its efficacy in annihilation of males of $Z$. tau while protein bait was most effective.

\section{Acknowledgements}

This work was supported by Dr. Rajendra Prasad Central Agriculture University and thanks to Dr. M.L. Agarwal for guidance during this work.

\section{References}

Agarwal, M.L. 2019. A checklist of Indian fruit flies (Diptera: Tephritidae) with notes on some species. J. Bombay Nat. Hist. Soc. (in press).

Bharathi, T.E., Sathiyanandam, V.K.R. and David, P.M.M. 2004. Attractiveness of some food baits to the melon fruit fly, Bactrocera cucurbitae (Coquillett) (Diptera: Tephritidae). Int. J. Tropical Insect Sci. 24(2): 125-134.

Boller, E.F. 1983. Biotechnical methods for the management of fruit fly populations. In: Fruit Flies of Economic Importance. Cavalloro, R. (ed.). CEC/IOBC Symp. Athens, 1982. Balkema, Rotterdam, pp. 342-352.

Doorenweerd, C., Leblanc, L., Norrbom, A.L., San Jose, M. and Rubinoff, D. 2018.A global checklist of the 932 fruit fly species in the tribe Dacini (Diptera, Tephritidae). ZooKeys., 730: 17-54.

Duyck, P.F., Quilici, S., Fabre, F. and Ryckewaert. P. 2004. Comparison and optimization of the efficacy of different food attractants for both sexes of the melon fly, Bactrocera cucurbitae (Coquillett) (Diptera: Tephritidae) In: Proceedings of the 6th International Symposium on Fruit Flies of Economic Importance. Barnes Brian N. (ed.). 6-10 May 2002, Stellenbosch, South Africa. Irene: Isteg Scientific Publications, pp. 351-354.

Ekesi, S. 2016. Baiting and male annihilation techniques for fruit fly suppression in Africa. In: Fruit fly Research and Development in Africa-Towards Sustainable Management Strategy to improve Horticulture. Ekesi, S., Mohamed, S.A. and De Meyer, M. (eds.), Springer, Switzerland, pp. 275292.

Hasyim, A., Muryati, Khairul, M., Riska and de Kogel, W.J. 2004. Fruit fly control in Indonesia. Annual Report, Research Institute of Fruit Solok. Unpublished Report (In Bahasa Indonesia).

Jhala, R.C. and Sisodiya, D.B. 2008. Male annihilation and bait application based management strategy for fruit flies. Bactrocera cucurbitae (Coq.) and Dacus ciliatus (Loew) (Tephritidae: Diptera) infesting cucurbit crops. In: Emerging Trend of Research in Insect Pest Management and Environmental Safety. Goel, S.C. (ed.). Uttar Pradesh Zoological Society (Publisher), Muzaffarnagar, India. 1: 133-140.

Kumar, B. and Agarwal, M.L. 2005b. Efficacy of different attractant and bait combinations against Bactrocera cucurbitae (Coquillett). Indian J. Plant Prot. 33(2): 194-196.

Thakur, M. and Gupta, D. 2013. Efficacy of different food attractants for control of fruit flies [Bactrocera tau and $B$. cucurbitae (Diptera : Tephritidae)]. Int. J. Agricult. Stat. Sci. 9(2): 575-580.

Vargas, R.I., Piñero, J.C. and Leblanc, L. 
2015. An overview of pest species of Bactrocera fruit flies (Diptera: Tephritidae) and the integration of biopesticides with other biological approaches for their management with a focus on the Pacific region. Insects., 6(2): 297-318.

How to cite this article:

Vasudha, A., M. L. Agarwal and Sreedhar, M. 2020. Efficacy of Different Bait and Attractant Combinations in Attracting Pumpkin Fruit Fly Zeugodacus (Zeugoodacus) Tau (Walker) (Diptera: Tephritidae). Int.J.Curr.Microbiol.App.Sci. 9(04): 2122-2130. doi: https://doi.org/10.20546/ijcmas.2020.904.255 